\title{
Supernovae and Molecular Clouds in Spiral Galaxies
}

\author{
Guo-xuan Song \\ Shanghai Astronomical Observatory, Chinese Academy of Sciences, \\ Shanghai, National Astronomical Observatories, China
}

\begin{abstract}
We report N-body simulations of the birthrate and distribution of supernovae in spiral galaxies. The simulations assume that stars form in GMCs with mass greater than $10^{5} M_{\odot}$ and that a Miller-Scalo IMF results. We assume that the resulting supernovae disrupt the GMC into smaller clouds; these clouds aggregrate to form new GMCs via inelastic collisions. Imposing a spiral potential, we find that supernovae form throughout the disk, but concentrated in the spiral arms. Using conditions appropriate for the Galaxy, a set of simulations with different initial random distributions of molecular clouds predicts $755 \tau_{5}$ supernova remnants (SNR) should exist in the Galaxy (where $\tau_{5}$ is the ratio of the lifetimes of SNRs to $10^{5} \mathrm{yr}$ ). The predicted number of remnants and their spatial distribution can be compared to observations.
\end{abstract}

\section{Introduction}

Supernova remnants are formed when stars with a certain masses end their lives in supernova explosions. Stars are born mainly in molecular clouds, especially in Giant Molecular Clouds (GMCs). Winds and expanding HII regions from the stars and, particularly, from supernova explosions, cause GMCs to fragment into less massive clouds and new GMCs are in turn formed by collisions between the less massive clouds. A thorough understanding of the cycle evolution of molecular clouds will further our understanding of the occurrence of supernova events.

Great effort has been put into the study of supernova events in our Galaxy as it involves not only the late stage of stellar evolution, but also the chemical evolution of metallicity.

Supernovae are divided into Type I and II. It is generally accepted that massive stars are the progenitors of Type II supernovae while Type I supernovae are produced in some case by carbon deflagration in C-O white dwarfs in binaries, and in other case, by other means(Chiappin, Mattencci \& Graten 1997). There is no consensus on the birthrate of the two types. Tammann(1982) stated that the rates are equal, while van den Bergh(1988) holds that the birthrate for Type II supernovae is about 3 times greater than for Type I. Certainly, further study of this question is needed. 


\section{Model}

The simulation begins with 120,000 molecular clouds each with $10^{4} M_{\odot}$, which are randomly distributed in the volume with scale height $100 \mathrm{pc}$ and radii between $3 \mathrm{kpc}$ and $7 \mathrm{kpc}$. They are acted upon by the fixed disk potential with a spiral perturbation as well as by the self-gravitation among the clouds. Clouds will aggregate to form more massive clouds by the inelastic collision among them. For aggregated clouds with mass greater than $10^{5} M_{\odot}$, star formation will take place within them. Formed high mass stars will end their short lifetime to induce supernova explosion. From this simulation the count and distribution of supernova remnants could be derived.

In the simulation a GMC will make stars of various masses depending on the probability function, which is proportional to the logarithm of its mass. When a GMC is identified to have star formation, the star formation efficiency is taken as 0.3 , i.e., about a third of mass of this GMC will turn to stars.

\section{Results}

From the numerical simulation, we find the number of stars with masses between 8 and $100 M_{\odot}$ formed per year assuming a Miller \& Scalo(1979) IMF is $7.6027 \times$ $10^{-3}$.

If Type II supernova events are the end-products of these stars, then the number is also the number of Type II supernova events per year. As the lifetime of supernovae is generally less than $10^{5} \mathrm{yr}$, let us write it as $10^{5} \tau_{5}$, where $\tau_{5}$ is a fudge factor defined as the ratio of a SNR lifetime to $10^{5} \mathrm{yr}$. Then there should be $780 \tau_{5}$ supernova remnants in our Galaxy.

From repeated simulations with different random initial distributions of clouds it is found that the number of supernova remnants in our Galaxy is $775 \tau_{5}$

\section{References}

Chiappin, C., Mattencci, F., \& Graten, R. 1997, ApJ 477, 765

Miller, G. E., \& Scalo, J. M. 1979, ApJS 41, 513

Tammann, G. A. 1982 In supernovae: A survey of current research eds. M. J. Rees \& R. J. Stonehouse(Dordrecht: Reidel), 371

van den Bergh, S. 1988, Comments on Astrophysics 12, 131 